\title{
Hausarzt sei wachsam: Präoperative Anämie als Prognosemerkmal
}

\section{Auch bei nicht kardialen Operationen ist eine leichte präoperative Anämie mit einem erhöhten Risiko für die postoperative 30-Tage-Morbidität und -Mortalität assoziiert.}

- Von Operationen am Herzen her ist bekannt, dass eine präoperative Anämie mit einer schlechteren Prognose nach der Operation einhergeht. Nun hat man anhand der Datenbank des Qualitätssicherungssystems des American College of Surgeons untersucht, ob das auch für Operationen außerhalb des Herzens zutrifft. Dabei wurden Daten des Jahres 2008 von 211 Krankenhäusern und insgesamt 227425 Patienten untersucht, von denen 69229 (30,44\%) präoperativ eine Anämie aufwiesen. $\mathrm{Zu}$ Grunde gelegt wurde dabei die letzte HämatokritBestimmung vor der Indexoperation, wobei nach den WHO-Kriterien ein Hämatokrit von unter $36,0 \%$ bei Frauen und von unter $39,0 \%$ bei Männern als Anämie definiert war.

Bei 97,99\% der Patienten lag ein Hämatokritwert aus dem Zeitraum von zwei Monaten vor der Operation vor, bei $92,83 \%$ innerhalb von vier Wochen und bei $80,99 \%$ innerhalb von zwei Wochen. Nach den Definitionskriterien wiesen 30,44\% der Patienten eine Anämie auf, davon $83,59 \%$ eine leichte und $16,41 \%$ eine mäßiggradige bis schwere Anämie. Nach Korrektur für konfundierende Faktoren lag die postoperative 30-Tagesmortalität bei den Patienten mit Anämie um $42 \%$ höher als bei denen ohne Anämie. Selbst beim Vorliegen einer nur leichten Anämie erhöhte sich die postoperative Mortalität bereits um $41 \%$ und stieg in der Gruppe mit mäßiggradiger bis schwerer Anämie auf $44 \%$ an. Ein ähnlicher Trend zeigte sich auch bei der postoperativen 30-Tage-Morbidität, die insgesamt um 35\% erhöht war. Postoperative Komplikationen machten sich bei den Patienten mit leichter Anämie mit einer Rate von $31 \%$ bemerkbar, bei denen mit mittelgradiger

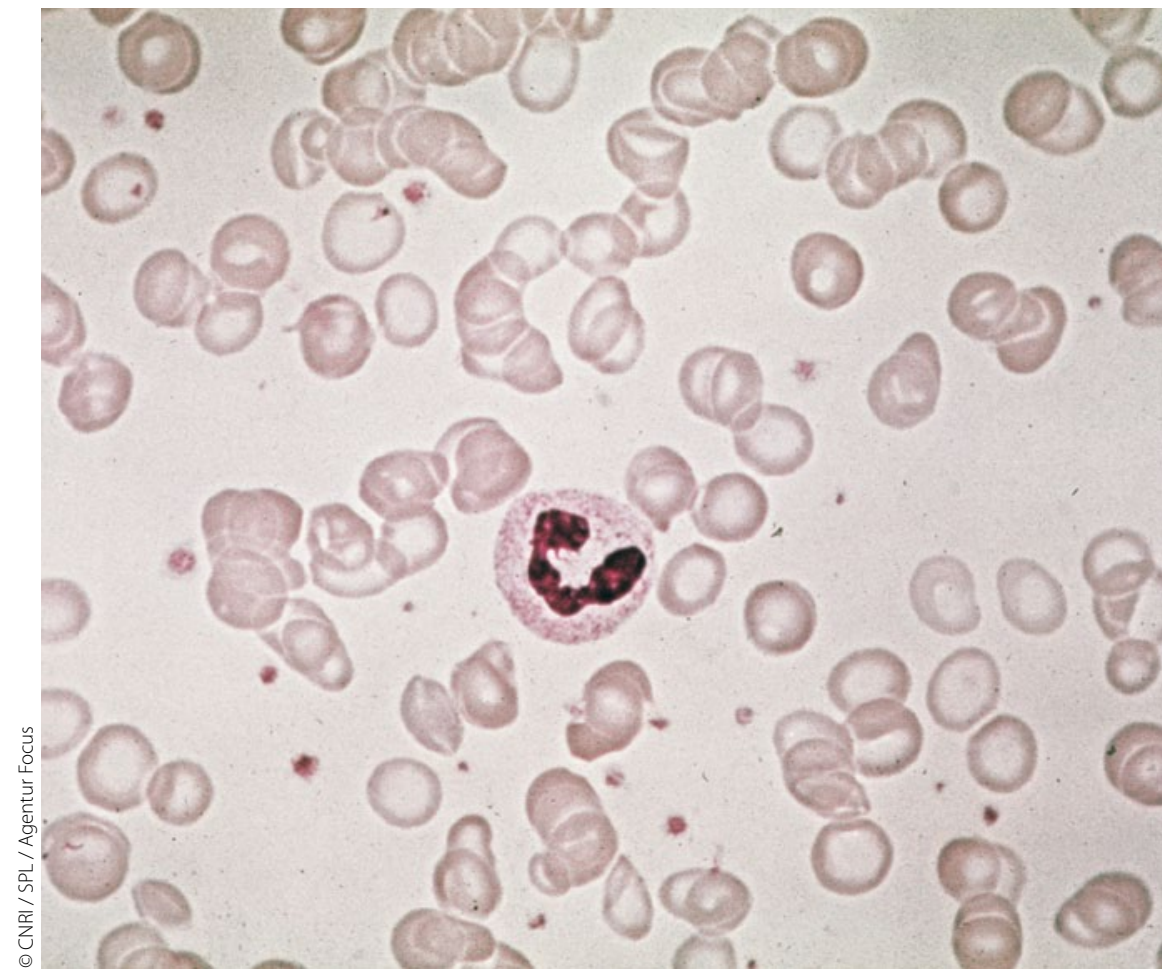

Hypochrome Anämie: Postoperative Mortalität erhöht.

bis schwerer Anämie sogar mit einer Rate von $56 \%$.

Beim Vergleich von Patienten ohne Anämie, jedoch mit einem definierten bekannten Risikofaktor schlug die Anämie für das postoperative Risiko stärker zu Buche als spezifische kardiale, respiratorische, renale Komorbiditäten, aber auch als lokale Wundprobleme, Sepsis oder thrombembolische Komplikationen. Bekamen Patienten perioperativ Bluttransfusionen, war dies erwartungsgemäß mit einer erhöhten Mortalitäts- und Morbiditätsrate assoziiert. Die Notwendigkeit einer perioperativen Bluttransfusion scheint sogar das Risiko einer präoperativen Anämie in den Hintergrund zu drängen. Erhielten Patienten perioperativ Transfusionen, war ihr postoperatives Risiko in jedem Fall erhöht und es bestand kein Unterschied zwischen den Untergruppen mit und ohne präoperative Anämie.
- K. Musallam et al. (Korres.: Dr. F. R. Jamali, Department of Surgery, American University of Beirut Medical Center, PO Box 11-0236, Beirut 1107 2020, Lebanon; fj03@aub.edu.lb): Praeoperative anaemia and postoperative outcomes in non-cardiac surgery: a retrospective cohort study. Lancet 378 (2011) 9800, 1396-1407

\section{Kommentar}

Der ungünstige Einfluss der Anämie auf die Prognose von Patienten nach Operationen wird sicher bisher zu wenig berücksichtigt. Gerade bei elektiven Eingriffen wächst hier dem Hausarzt eine wichtige Aufgabe zu, da vom Krankenhaus häufig erwartet wird, dass er die präoperativen Untersuchungen veranlasst. Leichtgradige Anämien werden nicht selten ignoriert, obwohl sie, wie die Untersuchung zeigt, durchaus negative Einflüsse auf den postoperativen Verlauf haben können.

H. S. FÜESSL - 